\title{
Competing interests in development of clinical practice guidelines for diabetes management: Report from a multidisciplinary workshop
}

\author{
Anna M Sawka' \\ Lilian Magalhães ${ }^{2}$ \\ Amiram Gafni $i^{3,4}$ \\ Gary F Lewis' \\ 'Division of Endocrinology, \\ Department of Medicine, University \\ Health Network and University \\ of Toronto, Toronto, Ontario, \\ Canada; ${ }^{2}$ School of Occupational \\ Therapy, University of Western \\ Ontario, London, Ontario, Canada; \\ ${ }^{3}$ Department of Clinical Epidemiology \\ and Biostatistics, McMaster University, \\ Hamilton, Ontario, Canada; ${ }^{4}$ Centre \\ for Health Economics and Policy \\ Analysis, Hamilton, Ontario, Canada
}

\begin{abstract}
Objective: To explore the complex issue of competing interests (CIs) in development of clinical practice guidelines (CPGs) in diabetes with stakeholders.

Methods: A multidisciplinary panel of 26 health, methodological, legal, and bioethical experts, trainees, and lay people from across Canada participated in a workshop on CIs in CPGs. Mixed methods were used such that qualitative themes were extracted from the discussions and quantitative survey data were collected.

Results: In the discussions, participants acknowledged that potential competing interests were not uncommon among sponsoring organizations and authors of CPGs. Avoidance of all potential CIs in development of CPGs was emulated as ideal, but considered probably unrealistic, given the paucity of peer-reviewed funding opportunities for development of evidence-informed CPGs and the scarcity of knowledgeable authors without CIs. An optimal approach for management of CIs in CPGs could not be agreed upon by participants. Full disclosure of any financial CIs for authors and sponsoring organizations as well as discouragement of external financial contributors from writing involvement, were endorsed by participants in the workshop and a subsequent survey.

Conclusions: Complete disclosure of financial CIs of sponsoring organizations and authors of CPGs is essential, yet the optimal approach to management of potential CIs is currently undefined.
\end{abstract}

Keywords: conflicts of interest, clinical practice guidelines, diabetes, knowledge translation, bioethics

\section{Introduction}

Serious concerns about competing interests (CIs) in diabetes-related clinical practice guideline (CPG) development have recently been voiced in the medical literature (Editorial [Canadian Medical Association Journal] 2005). Although CIs may take many forms, financial relationships of clinicians, scientists, and professional or diseaserelated organizations with industry have been the main focus of recent literature. In Canada, in the field of diabetes, the presence of undisclosed potential CIs of the authors of a CPG sponsored by the Canadian Diabetes Association was criticized in recent years (Editorial 2005). The specific criticism (Editorial 2005) stemmed from differences in recommendations on the use of a particular insulin formulation in the Canadian Diabetes Association (CDA) clinical practice guideline (Canadian Diabetes Association Clinical Practice Guidelines Expert Committee 2003), compared with those of a national drug formulary (Common Drug Review) (Canadian Coordinating Office for Health Technology Assessment 2005). The implication of the criticism was that undisclosed CIs of authors of the CDA guideline could potentially account for some of the differences in recommendations observed.
Toronto General Hospital, 200 Elizabeth Street, 12 EN-2I2, Toronto, Ontario, Canada, M5G 2C4

Email sawkaam@yahoo.com 
Relationships between industry and the authors of CPGs or specialty medical organizations are not uncommon, and may be under-reported. In a review of clinical guidelines published in six major medical journals in 1979, 1984, 1989, 1994, and 1999, only $3.7 \%$ of guidelines mentioned CIs (Papanikolaou et al 2001). Furthermore, in a crosssectional survey of 192 authors of 44 CPGs endorsed by North American and European societies on common adult diseases published between 1991 and 1999, an average of $81 \%$ of authors per clinical practice guideline had interactions with industry and the average author interacted with 10.5 different companies (Choudhry et al 2002). In a survey of Australian medical organizations, $59 \%$ of organizations reported receiving support from one or more pharmaceutical companies (Kerridge et al 2005). In a review of 200 guidelines deposited in the US National Guideline Clearinghouse in 2004, investigators from the journal, Nature, found that $35 \%$ of authors disclosed a conflict of interest, $16 \%$ of authors helped write guidelines on illnesses relevant to companies in which they owned stock, and $49 \%$ of guidelines did not include any details of authors' CIs (Taylor and Giles 2005). Moreover, an editor of the Journal of the American Medical Association has stated that, "drug company sponsors see guideline-issuing bodies as perfect places to exert influence" (Taylor and Giles 2005). Such criticisms have led to questions such as "can we trust the evidence in evidence-based medicine?", pertaining specifically to evidence-based guideline development (Abramson and Starfield 2005).

Our aim was to assemble a multidisciplinary, multiinstitutional, panel of experts, students, and lay people from across Canada to explore the issue of CIs in development and dissemination of CPGs, particularly focusing on diabetes care. The participants were identified as stakeholders in this issue, because of their clinical, academic, or methodologic focus, or because they acted as representatives of individuals with diabetes. Mixed methods, including qualitative and quantitative approaches were used in analyzing data that was collected.

\section{Methods}

\section{Format of the workshop and qualitative methodology}

A half-day workshop was held in Toronto, Canada on October 18, 2006. We invited health, methodological, legal, and bioethical experts, trainees, and lay people from across Canada. Four structured lectures 20-30 minutes in duration were each followed by open discussion periods, moderated by two investigators (AMS and AG). The proceedings were audio recorded, with the exception of one lecture, as per the request of the speaker. The session was closed to the public. All participants were informed that their personal views would not be identified outside the group and open discussion was encouraged. When necessary, the discussions were refocused onto relevant concepts, with acknowledgement of the presence of some conflicting views.

An approach founded in Delphi methodology was used in developing summary statements (Jones and Hunter 1995). A strict consensus was not the goal, given the sensitivity of the topic and the known conflicting views of some of the selected participants. A qualitative researcher (LM) and a research assistant took notes during the session, to supplement audiorecordings of the sessions. Relevant themes from the discussions and notes were extracted by the qualitative researcher (LM). The final theme summary statements were compiled by two of the investigators (LM and AS) using the data extracted by the qualitative researcher in her structured notes.

\section{Survey several months after the workshop}

A few months after the session, a self-administered survey summarizing key themes from the discussions was distributed to participants via electronic mail. In the questionnaire, summary statements were accompanied by a Likert scale, framed on a disagree - agree continuum (Streiner and Norman 1995), on a scale of 1 to 7 (with the value of 7 representing the strongest agreement). We defined "general strong agreement" with statements by a value of 5 or greater out of seven in all participants. "Opinion dissent" was reflected by agreement scores ranging from $\leq 3$ to $\geq 4$ among participants. "Some agreement" was defined by all agreement scores ranging from 4 to 7 among all participants. The means and standard deviations and ranges for agreement to Likert scale questions were calculated (SPSS 12.0; SPSS Inc., Chicago, IL). Completed final questionnaires were returned via electronic mail or fax as per respondent preference.

\section{Results}

\section{Description of participants}

\section{and the framework of the discussions}

Twenty-six participants in the workshop included experts in: health (diabetes and other diseases), methodology (epidemiology, knowledge synthesis, knowledge translation, 
biostatistics, and health economics), medical writing, health law, bioethics, and administration (related to a disease organization). Health experts were from multiple areas including endocrinology, medicine, primary care, ophthalmology, ethics, nursing, and nutrition. Authors of clinical practice guidelines (in diabetes and other diseases) participated. A patient representative and trainees in medicine-related specialties or ethics also participated. Representatives of the CDA as well as other disease organizations sponsoring clinical practice guidelines were included. Individuals with and without relationships with industry were included.

\section{Qualitative theme \#I: Multifaceted potential competing interests \\ and disclosure}

The existence of CIs among sponsoring organizations and authors of CPGs were recognized to be common. Some participants point out that, potential CIs could be multifaceted, including financial, academic, political, religious, cultural, personal, professional, or other organizational interests. The potential importance of nonfinancial CIs was stressed by some participants. The identified sources of financial CIs were thought to include industry, governments, third party payers, or other organizations. The optimal term for description of competing interests could not be agreed upon, with a few participants preferring the term, "duality of interest." Disclosure was used to describe the process of declaring potential CIs. The importance of disclosure of any financial CIs of sponsoring organizations or authors in the planning and development stages of CPGs was strongly asserted to be important by multiple participants. Furthermore, several participants stressed the importance of publicly disclosing CIs related to sponsoring organizations of CPGs and their authors. The lack of public disclosure of all financial CIs of sponsoring organizations and authors of CPGs was considered a threat to professional credibility and public trust in the CPG by some participants.

\section{Qualitative theme \#2: The importance of putting the evidence first in CPG recommendations}

Multiple participants asserted that CPG recommendations should be evidence-based, although it was acknowledged that some primary research evidence may be prone to CIs. The use of systematic techniques in reviewing and appraising existing evidence was encouraged by some participants.
The incorporation of economic analyses in all CPGs was not thought to be feasible, because of lack of human and financial resources to carry out such analyses properly and regional variability in costs, affordability, and values. The great financial cost of producing high quality, evidence-informed CPGs was stressed my individuals involved in development of such guidelines as well as representatives of sponsoring organizations.

\section{Qualitative theme \#3: Can competing interests be avoided in development of clinical practice guidelines?}

Some participants suggested that all potential financial CIs should ideally be avoided in development of CPGs. Concerns were expressed on the potential influence of relationships of physicians and the pharmaceutical industry, consciously or unconsciously, impacting recommendations. However, most participants conceded that avoidance of all financial CIs of sponsoring organizations or authors of CPGs, although ideal, may not be feasible at this time. The high need for financial and human resources in developing and disseminating high quality CPGs and the lack of peer-reviewed independent funding for such purposes, were acknowledged as important challenges. Furthermore, the contribution of knowledge and expertise from authors with known CIs, was highlighted by several participants. The scarcity of clinical content or methodologic experts without CIs was asserted. Thus, strict exclusion of all experts with CIs from CPG development could not be endorsed by most members of the group. This issue was subject to some opinion debate. However, independence of authorship and writing editing of a guideline, without any direct input of financial supporters was strongly supported by multiple participants.

\section{Quantitative survey data}

A survey was distributed to participants several months after the workshop. In this survey, issues that were discussed at the session were summarized in the form of summary statements. The response rate to this self-administered survey was $57.7 \%$ $(15 / 26)$. A summary of the responses is shown in Table 1 . We observed strong general agreement with statements relating to the definition of disclosure and the suggestion that CIs should be declared by sponsoring organizations and authors in the planning stage of a CPG. There was some agreement with statements suggesting that the development of CPGs is important and should not be abandoned as a knowledge 
Table I Results of an opinion survey several months after the workshop

Statement (number of responses, maximum I5)

\section{Statement (number of responses, maximum I5)}

I.The term "competing interests" reasonably reflects

various potential multifaceted interests of organizations

or authors of clinical practice guidelines. (I5)

2. Competing interests may include financial, academic, political, cultural, religious, or other personal or organizational interests related to a topic. (15)

3. Disclosure is the process of declaring competing interests. (15)

4. The presence of undisclosed, unaddressed, competing interests may threaten: a) the credibility of clinical practice guidelines among healthcare professionals and b) the public's trust. (15)

5. Ideally, in the planning of a clinical practice guideline, a dedicated (internal or external) advisory panel should be established to evaluate, monitor, record, and ultimately disclose any competing interests related to the guideline or its authors. (I5)

6. Competing interests should be declared by sponsoring organizations and authors in the planning stage of a clinical practice guideline. (15)

7. Contributing authors of a clinical practice guideline should be informed of the competing interests of the sponsoring organization as well as other coauthors in the planning stage of the guideline. (I5)

8. Disclosure of competing interests related to clinical practice guidelines should be made public upon release of the guideline. $(14)^{c}$

9. Ideally, the presence of competing interests should be minimized for clinical practical guidelines, but complete avoidance of all potential conflicts of interests for sponsoring organizations and authors may not always be possible. (15)

10. If there are external financial contributors for a clinical practice guideline, such supporters should not be involved in any aspect of writing of the guideline. (15)

II. Our group did not reach consensus on a threshold at which a contributing author has excessive competing interests precluding involvement in development of a clinical practice guideline. (15)

12. Recommendations in clinical practice guidelines should be evidence-based, although it is acknowledged that some primary research evidence may prone to competing interests. (15)

13. Although the inclusion of economic analyses exploring the cost of recommendations of clinical practice guidelines may be ideal, it may not be currently feasible for all clinical practice guidelines because of lack of human and financial resources to carry out such analyses properly as well as regional variability in costs and values that might affect affordability of recommendations. (15)
Likert scale agreement ${ }^{a}$

Mean (Standard deviation)

(Range of values)

$\begin{array}{ll}4.9(1.5) & \text { Opinion dissent } \\ (2,7) & \end{array}$

6.1 ( 1.3$)$

Opinion dissent

$(2,7)$

$6.5(0.6)$

General strong agreement

$(5,7)$

6.1 (I.3)

Opinion dissent

$(3,7)$

$4.9(1.6)$

$(2,7)$

Opinion dissent

$6.6(0.6)$

General strong agreement

$(6,7)$

$6.3(0.9)$

Some agreement

$(4,7)$

$6.4(0.9)$

Some agreement

$(4,7)$

5.5 ( 1.8$)$

Opinion dissent

$(I, 7)$

$6.6(0.9)$

Some agreement

$(4,7)$

$6.0(0.9)$

Some agreement

5.7 ( I.0)

Some agreement

$(4,7)$

$5.9(0.9)$

$(4,7)$
Some agreement 
14. Future research is needed to develop innovative strategies to deal with competing interests in clinical practice guideline development. (15)

15. Future research is needed to develop effective knowledge translation strategies for dissemination and accurate representation of clinical practice guideline recommendations. (15)

16. The development of clinical practice guidelines is important and should not be abandoned as a knowledge translation strategy aimed at healthcare professionals. (15)
$5.6(1.6)$

$(3,7)$

$6.3(0.9)$

$(4,7)$

$6.6(1.1)$

$(4,7)$
Opinion dissent

Some agreement

Some agreement

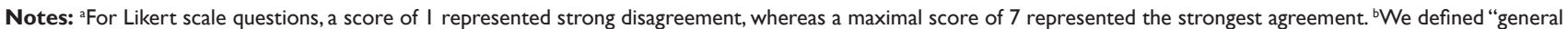
strong agreement" with statements by a value of 5 or greater out of seven in all participants. "Opinion dissent" was reflected by agreement scores ranging from $\leq 3$ to $\geq 4$ among participants. "Some agreement" was defined by all agreement scores ranging from 4 to 7 among all participants. 'One blank response.

translation strategy aimed at healthcare professionals. Some agreement was also observed with a suggestion that research needs to be aimed at developing effective knowledge translation strategies for dissemination and accurate representation of CPG recommendations. Respondents generally agreed that CPG recommendations should be evidence-based, without the absolute requirement of economic analyses. There was some agreement with statements suggesting that financial contributors be excluded from any aspect of writing of a CPG and that disclosure of CIs be made public. Opinion dissent was expressed with respect to the following issues: competing interests terminology, the multifaceted composition of CIs, the impact of CIs on the credibility of a CPG, and public trust, the suggestion of an advisory panel monitoring CPGs for sponsoring organizations, and the feasibility of strict avoidance of CIs.

\section{Discussion}

We assembled a Canadian multidisciplinary panel and explored the concept of competing interests in development and dissemination of CPGs. Our findings highlighted the complexity and sensitivity of stakeholders to his issue. The need for transparency and disclosure of CIs of sponsoring organizations and authors of CPGs were highlighted in the qualitative and quantitative data collected. However, considerable opinion dissent was observed with respect to the issues of threshold of acceptability of CIs for authors and sponsoring organizations writing CPGs and the impact of CIs on the credibility of recommendations. Concerns were expressed on the potential influence of relationships of physicians and the pharmaceutical industry, consciously or unconsciously, impacting recommendations. It is important to acknowledge that in a prior review paper, Wazana (2000) reported that physician meetings with pharmaceutical representatives were associated with changes in drug formularies and prescribing behavior. However, evidencebased approaches to developing CPG recommendations were favored in CPG development by our group in hopes of minimizing bias. Yet, the use of such approaches may not necessarily safeguard CPGs from the influence of CIs, as primary research and its interpretation may be subject to potential influences of CIs. For example, it has been reported in the literature that industry sponsorship of studies is associated with pro-industry conclusions (Bekelman et al 2003). An optimal, feasible approach to minimizing the impact of CIs on CPG development could not be agreed upon in our group. In the interim, transparency of process and full disclosure of CIs were deemed essential for the credibility of CPGs

The strengths of our workshop findings are that we were able to assemble a multidisciplinary panel of experts, trainees, and a lay person from a variety of backgrounds to openly discuss a highly complex, sensitive topic that influences clinical care in diabetes care and other conditions. Furthermore, we were able to secure a closed environment, stimulating frank (and at times heated) debate among individuals with a variety of perspectives. Limitations of our study included: the lack of any strict consensus recommendations, a limited response rate to our post-workshop questionnaire, and the lack of international participants. We suspect that the relatively low response rate to our post-workshop questionnaire may be due to the sensitivity of the topic.

In terms of future research directions, it would be instructive for a multidisciplinary panel of stakeholders involved in management of other disease conditions, to explore opinions on the issue of CIs in CPGs. Also, qualitative research exploring the opinions of lay people on this issue would be 
valuable. In the mean time, core values including, an ethically conscious, evidence-informed, transparent approach in the development of CPGs has been emphasized in our study, in absence of any strict consensus on the threshold of acceptance (or rejection) for CIs in CPGs.

\section{Acknowledgments}

The authors are indebted to the speakers and participants of this workshop who contributed their time and opinions to this project. This project was funded by workshop support from the Canadian Institutes of Health Research (Institute of Nutrition, Metabolism, and Diabetes). The authors like to thank Ms. Lucy Montana for assisting us with the arrangements for the workshop. The authors would also like to thank Dr. Jeremy Gilbert, Shamila Kamalanathan, Dr. Siarhei Slizeuski, and Dr. Shahryar Murshed for assistance with preparing for the workshop as well as registration of participants.

\section{Disclosure}

This workshop was funded by the Canadian Institutes of Health Research (Institute of Nutrition, Metabolism, and Diabetes), Grant \# OWK-79938. Anna M Sawka has previously been awarded a Health Economics Fellowship from McMaster University that was funded in part by HoffmanLaRoche, Ltd. Lilian Magalhães declares no conflicts of interest. Amiram Gafni has consulted to various companies and governments and has received grant support from both government and industry. Gary F Lewis has consulted for Merck Frosst, Pfizer, Eli Lilly, Scherring, Astra Zeneca and has received grant support from Merck Frosst and Amylin Pharmaceuticals.

\section{References}

Abramson J, Starfield B. 2005. The effect of conflict of interest on biomedical research and clinical practice guidelines: Can we trust the evidence in evidence-based medicine? JABFP, 18:414-18.

Bekelman JE, Li Y, Gross CP. 2003. Scope and impact of financial conflicts of interest in biomedical research. JAMA, 289:454-65.

Canadian Coordinating Office for Health Technology Assessment. 2005. CEDAC final recommendation on reconsideration and reasons for recommendation. Insulin glargine (Lantus ${ }^{\circledR}-$ Aventis Pharma Inc.) [online]. Accessed November 24, 2005. URL: http://www.ccohta.ca/CDR/cdr_pdf/cdr_submissions/Complete/ cdr_complete_Lantus_2005Sept28.pdf.

Canadian Diabetes Association Clinical Practice Guidelines Expert Committee; Canadian Diabetes Association. 2003. Clinical practice guidelines for the prevention and management of diabetes in Canada. Can $J$ Diabetes, 27(suppl 2):S1-S152.

Choudhry NK, Stelfox HT, Detsky AS. 2002. Relationships between authors of clinical practice guidelines and the pharmaceutical industry. JAMA, 287:612-17.

Editorial. 2005. Clinical practice guidelines and conflicts of interest. CMAJ, 173:1297.

Jones J, Hunter D. 1995. Qualitative research: Consensus methods for medical and health services research. BMJ, 311:376-80.

Kerridge I, Maguire D, Newby D, et al. 2005. Cooperative partnerships or conflicts-of interest? A national survey of interaction between the pharmaceutical industry and medical organizations. Intern Med J, 35:206-10.

Papanikolaou GN, Baltogianni MS, Contopoulos-Ioannidis DG, et al. 2001. Reporting of conflicts of interest in guidelines of preventative and therapeutic interventions. BMC Med Res Methodol, 1:3.

Streiner DL, Norman GR. 1995. Chapter 4: Scaling responses. Health measurement scales: A practical guide to their development and use. 2nd ed. Oxford, UK: Oxford University Press, 34-8.

Taylor R, Giles J. 2005. Cash interests taint drug advice. Nature, 437:1070-1.

Wazana A. 2000. Physicians and the pharmaceutical industry. Is a gift ever just a gift? JAMA, 283:373-80. 